Supporting information for:

\title{
Contamination of Urban Stormwater Pond Sediments: A Study of 259 Legacy and Contemporary Organic Substances
}

Kelsey Flanagan, ${ }^{1}$ Godecke-Tobias Blecken, ${ }^{l}$ Heléne Österlund, ${ }^{l}$ Kerstin Nordqvist, ${ }^{1}$ Maria Viklander ${ }^{l}$

${ }^{1}$ Urban Water Engineering, Department of Civil, Environmental and Natural Resources Engineering, Luleå University of Technology, 97187 Luleå, Sweden

*Corresponding author:

kelsey.flanagan@Itu.se

26 pages; 11 tables; 3 figures 
I. Characteristics of studied facilities

\begin{tabular}{|c|c|c|c|c|c|c|c|c|c|}
\hline Facility & Municipality & Facility type & $\begin{array}{l}\text { Catchment } \\
\text { classification }\end{array}$ & $\begin{array}{c}\text { Catchment } \\
\text { imperviousness }\end{array}$ & $\begin{array}{c}\text { Catchment } \\
\text { surface area } \\
\text { (ha) }\end{array}$ & Type of inlet & $\begin{array}{c}\text { Year of } \\
\text { construction }\end{array}$ & $\begin{array}{c}\text { Facility } \\
\text { volume } \\
\text { (m3) }\end{array}$ & $\begin{array}{c}\text { Facility } \\
\text { surface area } \\
\text { (ha) }\end{array}$ \\
\hline$\ddot{O} s-1$ & Östersund & Pond & $\begin{array}{c}\text { Industrial / } \\
\text { commericial }\end{array}$ & 97 & 91 & Pipe & 2000 & 9100 & 0.45 \\
\hline$\ddot{O} r-1$ & Örebro & Pond & $\begin{array}{c}\text { Industrial / } \\
\text { commericial }\end{array}$ & 40 & 40 & Pipe & 1996 & 1300 & 0.13 \\
\hline$\ddot{O} r-2$ & Örebro & Pond & $\begin{array}{c}\text { Industrial / } \\
\text { commericial }\end{array}$ & 50 & 57 & $\begin{array}{c}\text { Open } \\
\text { channel }\end{array}$ & 2000 & ND & 0.48 \\
\hline$\ddot{O} r-3$ & Örebro & Pond & Residential & 22 & 1490 & Pipe & 2010 & 20500 & 1.78 \\
\hline$\ddot{O} r-4$ & Örebro & Pond & Residential & 5 & 14.5 & $\begin{array}{c}\text { Open } \\
\text { channel }\end{array}$ & 2007 & ND & 0.33 \\
\hline$\ddot{O} r-5$ & Örebro & Pond & $\begin{array}{l}\text { Industrial / } \\
\text { commericial }\end{array}$ & 80 & 30 & $\begin{array}{c}\text { Open } \\
\text { channel }\end{array}$ & 2006 & 5800 & 0.5 \\
\hline$\ddot{O} r-6$ & Örebro & Pond & $\begin{array}{c}\text { Industrial / } \\
\text { commericial }\end{array}$ & 75 & 31 & $\begin{array}{c}\text { Open } \\
\text { channel }\end{array}$ & 2006 & 5800 & 0.8 \\
\hline$S-1$ & Stockholm & Pond & $\begin{array}{c}\text { Industrial / } \\
\text { commericial }\end{array}$ & ND & 78 & Pipe & 2009 & 1450 & 0.17 \\
\hline$S-2$ & Stockholm & Pond & Residential & ND & 304 & Pipe & 2007 & 530 & 0.05 \\
\hline$S-3$ & Stockholm & Pond & Residential & ND & 770 & Pipe & 1997 & ND & 0.06 \\
\hline$S-4$ & Stockholm & Pond & $\begin{array}{c}\text { Road / } \\
\text { highway }\end{array}$ & 50 & 2 & Pipe & 2002 & 498 & 0.03 \\
\hline$S-5$ & Stockholm & Pond & $\begin{array}{l}\text { Road / } \\
\text { highway }\end{array}$ & ND & ND & ND & ND & ND & 0.06 \\
\hline$S-6$ & Stockholm & $\begin{array}{c}\text { Subsurface } \\
\text { sedimentation } \\
\text { tank }\end{array}$ & $\begin{array}{c}\text { Road / } \\
\text { highway }\end{array}$ & 100 & 1.14 & Pipe & 1997 & 132 & 0.006 \\
\hline$V-1$ & Växjö & Pond & $\begin{array}{l}\text { Industrial / } \\
\text { commericial }\end{array}$ & 46 & 519 & $\begin{array}{l}\text { Pipe / open } \\
\text { channel }\end{array}$ & 1989 & 3300 & 0.36 \\
\hline$V-2$ & Växjö & Pond & Residential & 30 & 69 & $\begin{array}{c}\text { Pipe / open } \\
\text { channel }\end{array}$ & 2008 & 6600 & 0.36 \\
\hline$V-3$ & Växjö & Pond & $\begin{array}{l}\text { Industrial / } \\
\text { commericial }\end{array}$ & 60 & 330 & $\begin{array}{l}\text { Pipe / open } \\
\text { channel }\end{array}$ & 1994 & 17600 & 1.1 \\
\hline$V-4$ & Växjö & Pond & $\begin{array}{l}\text { Industrial / } \\
\text { commericial }\end{array}$ & 54 & 7.5 & Pipe & 1988 & 870 & 0.06 \\
\hline
\end{tabular}

Table S1. Characteristics of studied facilities. ND means that data was unavailable for a given site. 
II. Frequency of quantification of all substances

\begin{tabular}{|c|c|c|c|c|}
\hline Frequency & \multicolumn{4}{|c|}{ Substances } \\
\hline \multirow{45}{*}{$\begin{array}{l}\text { Never } \\
\text { quantified }\end{array}$} & $\mathrm{C}_{5}-\mathrm{C}_{8}$ Aliphatics & Styrene & Dimoxystrobin & Methiocarb \\
\hline & $\mathrm{C}_{8}-\mathrm{C}_{10}$ Aliphatics & MTBE & Diuron & sulfoxide \\
\hline & Nap & Aldrin & Epoxiconazole & Metconazole \\
\hline & Acyl & Dieldrin & Fenoxycarb & Metolachlor \\
\hline & Acen & Endrin & Fipronil & Methomyl \\
\hline & A & Isodrin & Fipronil sulfone & Methomyl \\
\hline & Dichloromethane & Telodrin & Fluazifop & oxime \\
\hline & 1,1-dichloroethane & alpha-HCH & Fonofos & Metribuzin \\
\hline & 1,2-dichloroethane & beta-HCH & Phorate & Oxamyl \\
\hline & 1,2-dichloropropane & Lindane & Phosalone & Pendimethalin \\
\hline & Chloroform & Heptachlor & Phosphamidon & Pethoxamid \\
\hline & Tetrachloromethane & cis-Heptachlor & Phosmet & Pirimicarb \\
\hline & Trans-1,2-dichloroethene & epoxide & Phosmet oxon & Prochloraz \\
\hline & 1,1,1-trichloroethane & trans-Heptachlor & Hexazinone & Prometon \\
\hline & Trichloroethene & epoxide & 2-hydroxyatrazine & Prometryn \\
\hline & Vinyl chloride & alpha-Endosulfan & Imidacloprid & Propazine \\
\hline & Monochlorobenzene & Simazine-2-hydroxy & Imidacloprid olefin & Propoxur \\
\hline & 1,2,3-trichlorobenzene & Carbofuran-3- & Imidacloprid urea & Pyrimethanil \\
\hline & 1,2,3,4-tetrachlorobenzene & hydroxy & Indoxacarb & Sebuthylazine \\
\hline & $1,2,3,5+1,2,4,5-$ & 6-chloronicotinic & Isoproturon & Simazine \\
\hline & tetrachlorobenzene & acid & Isoproturon-desmethyl & Simetryn \\
\hline & Diclobenil & Acetamiprid & Isoproturon- & Tebuconazole \\
\hline & Quintozene- & Acetochlor & monodesmethyl & Terbuthylazine \\
\hline & pentachloroaniline & Alachlor & Cadusafos & Thiacloprid \\
\hline & 2-monochlorophenol & Aldicarb & Carbaryl & Thiamethoxam \\
\hline & 3-monochlorophenol & Aldicarb sulfone & Carbofuran & Pentanedial \\
\hline & 4-monochlorophenol & Aldicarb sulfoxide & Clomazone & DeBB \\
\hline & 2,3-dichlorophenol & Ametryn & Chlorfenvinphos & HBCD \\
\hline & 2,4 +2,5-dichlorophenol & Atrazine & Chloridazon & PFHpA \\
\hline & 2,6-dichlorophenol & Azoxystrobin & Chloridazon-desphenyl & PFOA \\
\hline & 3,4-dichlorophenol & Boscalid & Chloridazon- & PFNA \\
\hline & 3,5-dichlorophenol & Cyanazine & methyldesphenyl & PFDA \\
\hline & 2,3,4-trichlorophenol & Cyproconazole & Chlorpyrifos & PFBS \\
\hline & 2,3,5-trichlorophenol & Atrazine-desethyl & Chlorsulfuron & PFHxS \\
\hline & 2,3,6-trichlorophenol & Terbuthylazine- & Chlortoluron & PFHpS \\
\hline & 2,4,5-trichlorophenol & desethyl & Clothiadinin & 6:2 FTS \\
\hline & 2,4,6-trichlorophenol & Atrazine- & Kresoxim-methyl & $8: 2$ FTS \\
\hline & 3,4,5-trichlorophenol & desisopropyl & Linuron & PFTrDA \\
\hline & 2,3,5,6-tetrachlorophenol & Desmetryn & Malaoxon & DEP \\
\hline & 2,3,4,5-tetrachlorophenol & Diazinon & Malathion & DNPP \\
\hline & 2,3,4,6-tetrachlorophenol & Difenacoum & Metamitron & DNHP \\
\hline & Pentachlorophenol & Diflufenican & Metazachlor & TetBT \\
\hline & Benzene & Dichlorvos & Methidathion & TCHT \\
\hline & Ethylbenzene & Dicrotophos & Methiocarb & TPhT \\
\hline & Xylene & Dimethoate & Methiocarb sulfone & \\
\hline \multirow{16}{*}{$0<\mathbf{f}_{\text {quant }} \leq \mathbf{2 5 \%}$} & $\mathrm{C}_{8}-\mathrm{C}_{10}$ Aromatics & Hexachlorobenzene & Propional & FOSA \\
\hline & $\mathrm{C}_{16}-\mathrm{C}_{35}$ Aromatics & Toluene & Butanal & PFTeDA \\
\hline & $\mathrm{F}$ & $o, p^{\prime}-\mathrm{DDT}$ & BDE 28 & MeFOSA \\
\hline & $\mathrm{BkF}$ & $\mathrm{p}, \mathrm{p}^{\prime}-\mathrm{DDT}$ & BDE 47 & EtFOSA \\
\hline & DahA & o,p'-DDD & BDE 99 & MeFOSE \\
\hline & Hexachloroethane & p,p'-DDD & BDE 100 & EtFOSE \\
\hline & Cis-1,2-dichloroethene & o,p'-DDE & BDE 153 & DMP \\
\hline & 1,1,2-trichloroethane & p,p'-DDE & BDE 154 & DPP \\
\hline & Tetrachloroethene & Terbuthylazine- & TBBP-A & DBP \\
\hline & 1,1-dichloroethene & desethyl-2- & PFBA & DiBP \\
\hline & 1,2-dichlorobenzene & hydroxy & PFPeA & DNOP \\
\hline & 1,3-dichlorobenzene & Hydroxy- & PFHxA & BBP \\
\hline & 1,4-dichlorobenzene & terbutylazine & PFUnDA & DCP \\
\hline & 1,2,4-trichlorobenzene & Carbendazim & PFDoDA & MPhT \\
\hline & 1,3,5-trichlorobenzene & Propiconazole & PFDS & DPhT \\
\hline & Pentachlorobenzene & Terbutryn & & \\
\hline
\end{tabular}




\begin{tabular}{|c|c|c|c|c|}
\hline Frequency & \multicolumn{4}{|c|}{ Substances } \\
\hline \multirow{5}{*}{$25<\mathbf{f}_{\text {quant }} \leq \mathbf{5 0} \%$} & $\mathrm{C}_{10}-\mathrm{C}_{12}$ Aliphatics & Chry & PCB 28 & DiDP \\
\hline & $\mathrm{C}_{10}-\mathrm{C}_{16}$ Aromatics & $\mathrm{BbF}$ & PFOS & DiNP \\
\hline & Phen & $\mathrm{BaP}$ & OP & TBT \\
\hline & Fluo & BPer & NP & MOT \\
\hline & $\mathrm{BaA}$ & IP & DBP & DOT \\
\hline \multirow{3}{*}{$50<f_{\text {quant }} \leq 75 \%$} & $\mathrm{C}_{12}-\mathrm{C}_{16}$ Aliphatics & PCB 101 & PCB 138 & MBT \\
\hline & Pyr & PCB 118 & PCB 180 & DBT \\
\hline & PCB 52 & PCB 153 & DEHP & \\
\hline $75<f_{\text {quant }}<100 \%$ & $\mathrm{C}_{16}-\mathrm{C}_{35}$ Aliphatics & Formaldehyde & Acetaldehyde & \\
\hline
\end{tabular}

Table S2: List of substances by frequency of quantification in stormwater pond sediments, $n=32$ 
III. Summary of quantified substances

\begin{tabular}{|c|c|c|c|c|c|}
\hline \multirow{2}{*}{ Substance group } & \multirow{2}{*}{ Substance } & \multirow{2}{*}{$\mathbf{f}_{\text {quant }}(\%)$} & \multicolumn{3}{|c|}{ Concentrations } \\
\hline & & & Median & Minimum & Maximum \\
\hline \multirow{7}{*}{ Hydrocarbons } & $\mathrm{C}_{10}-\mathrm{C}_{12}$ Aliphatics (mg/kg TS) & $41 \%$ & $<10$ & $<10$ & 146 \\
\hline & $\mathrm{C}_{12}-\mathrm{C}_{16}$ Aliphatics (mg/kg TS) & $53 \%$ & 11.5 & $<10$ & 285 \\
\hline & $\mathrm{C}_{5}-\mathrm{C}_{16}$ Aliphatics (mg/kg TS) & $53 \%$ & 18 & $<11$ & 410 \\
\hline & $\mathrm{C}_{16} \mathrm{C}_{35}$ Aliphatics (mg/kg TS) & $97 \%$ & 205 & $<10$ & 3820 \\
\hline & $\mathrm{C}_{8}-\mathrm{C}_{10}$ Aromatics (mg/kg TS) & $16 \%$ & $<0.48$ & $<0.095$ & 1.8 \\
\hline & $\mathrm{C}_{10}-\mathrm{C}_{16}$ Aromatics (mg/kg TS) & $28 \%$ & $<1.24$ & $<0.085$ & 1.24 \\
\hline & $\mathrm{C}_{16}-\mathrm{C}_{35}$ Aromatics (mg/kg TS) & $25 \%$ & $<1$ & $<1$ & 4.2 \\
\hline \multirow{17}{*}{$\begin{array}{l}\text { Polycyclic } \\
\text { aromatic } \\
\text { hydrocarbons } \\
\text { (PAHs) }\end{array}$} & $\mathrm{F}(\mathrm{mg} / \mathrm{kg} \mathrm{TS})$ & $6 \%$ & $<0.08$ & $<0.08$ & 0.095 \\
\hline & Phen (mg/kg TS) & $38 \%$ & $<0.08$ & $<0.08$ & 0.628 \\
\hline & Fluo $(\mathrm{mg} / \mathrm{kg} \mathrm{TS})$ & $50 \%$ & 0.0855 & $<0.08$ & 1.2 \\
\hline & Pyr (mg/kg TS) & $53 \%$ & 0.109 & $<0.08$ & 1.41 \\
\hline & $\mathrm{BaA}(\mathrm{mg} / \mathrm{kg} \mathrm{TS})$ & $38 \%$ & $<0.08$ & $<0.08$ & 0.464 \\
\hline & Chry (mg/kg TS) & $41 \%$ & $<0.08$ & $<0.08$ & 0.395 \\
\hline & $\mathrm{BbF}(\mathrm{mg} / \mathrm{kg} \mathrm{TS})$ & $50 \%$ & 0.0935 & $<0.08$ & 0.773 \\
\hline & $\mathrm{BkF}(\mathrm{mg} / \mathrm{kg} \mathrm{TS})$ & $25 \%$ & $<0.08$ & $<0.08$ & 0.179 \\
\hline & $\mathrm{BaP}(\mathrm{mg} / \mathrm{kg} \mathrm{TS})$ & $34 \%$ & $<0.08$ & $<0.08$ & 0.444 \\
\hline & DahA (mg/kg TS) & $22 \%$ & $<0.08$ & $<0.08$ & 0.13 \\
\hline & $\mathrm{BPer}(\mathrm{mg} / \mathrm{kg} \mathrm{TS})$ & $44 \%$ & $<0.08$ & $<0.08$ & 0.548 \\
\hline & IP (mg/kg TS) & $31 \%$ & $<0.08$ & $<0.08$ & 0.328 \\
\hline & Sum of $16 \mathrm{PAH}(\mathrm{mg} / \mathrm{kg} \mathrm{TS})$ & $53 \%$ & 0.64 & $<0.2$ & 6.4 \\
\hline & Carcinogenic PAH (mg/kg TS) & $50 \%$ & 0.28 & $<0.11$ & 2.6 \\
\hline & Sum other PAH (mg/kg TS) & $53 \%$ & 0.36 & $<0.092$ & 3.8 \\
\hline & Sum medium-weight PAH (mg/kg TS) & $53 \%$ & 0.215 & $<0.092$ & 3.2 \\
\hline & Sum high-weight PAH (mg/kg TS) & $50 \%$ & 0.32 & $<0.11$ & 3.2 \\
\hline BTEX & Toluene (mg/kg TS) & $13 \%$ & $<0.1$ & $<0.1$ & 1.28 \\
\hline \multirow{5}{*}{$\begin{array}{l}\text { Chlorinated } \\
\text { aliphatics }\end{array}$} & Hexachloroethane (mg/kg TS) & $3 \%$ & $<0.01$ & $<0.01$ & 0.126 \\
\hline & Cis-1,2-dichloroethene (mg/kg TS) & $3 \%$ & $<0.04$ & $<0.04$ & 0.18 \\
\hline & 1,1,2-trichloroethane $(\mathrm{mg} / \mathrm{kg} \mathrm{TS})$ & $3 \%$ & $<0.01$ & $<0.01$ & 0.125 \\
\hline & Tetrachloroethene (mg/kg TS) & $3 \%$ & $<0.02$ & $<0.02$ & 0.094 \\
\hline & 1,1-dichloroethene (mg/kg TS) & $3 \%$ & $<0.01$ & $<0.01$ & 0.167 \\
\hline \multirow{8}{*}{ Chlorobenzenes } & 1,2-dichlorobenzene $(\mathrm{mg} / \mathrm{kg} \mathrm{TS})$ & $3 \%$ & $<0.02$ & $<0.02$ & 0.69 \\
\hline & 1,3-dichlorobenzene (mg/kg TS) & $3 \%$ & $<0.02$ & $<0.02$ & 0.22 \\
\hline & 1,4-dichlorobenzene $\quad(\mathrm{mg} / \mathrm{kg}$ TS $)$ & $3 \%$ & $<0.02$ & $<0.02$ & 0.47 \\
\hline & 1,2,4-trichlorobenzene (mg/kg TS) & $3 \%$ & $<0.03$ & $<0.03$ & 0.31 \\
\hline & 1,3,5-trichlorobenzene $\quad(\mathrm{mg} / \mathrm{kg} \mathrm{TS})$ & $3 \%$ & $<0.05$ & $<0.05$ & 0.39 \\
\hline & Pentachlorobenzene (mg/kg TS) & $3 \%$ & $<0.01$ & $<0.01$ & 0.02 \\
\hline & $\begin{array}{l}\text { sum tetra- and pentachlorobenzenes }(\mathrm{mg} / \mathrm{kg} \\
\mathrm{TS})\end{array}$ & $3 \%$ & $<0.02$ & $<0.017$ & 0.04 \\
\hline & Hexachlorobenzene (mg/kg TS) & $9 \%$ & $<0.005$ & $<0.005$ & 0.168 \\
\hline \multirow{4}{*}{ Aldehydes } & Formaldehyde (mg/kg TS) & $97 \%$ & 1.2 & $<0.38$ & 8.4 \\
\hline & Acetaldehyde (mg/kg TS) & $88 \%$ & 0.86 & $<0.22$ & 3.6 \\
\hline & Propional (mg/kg TS) & $3 \%$ & $<0.2$ & $<0.2$ & 0.5 \\
\hline & Butanal (mg/kg TS) & $6 \%$ & $<0.2$ & $<0.2$ & 0.5 \\
\hline
\end{tabular}




\begin{tabular}{|c|c|c|c|c|c|}
\hline \multirow{2}{*}{ Substance group } & \multirow{2}{*}{ Substance } & \multirow{2}{*}{$\mathbf{f}_{\text {quant }}(\%)$} & \multicolumn{3}{|c|}{ Concentrations } \\
\hline & & & Median & Minimum & Maximum \\
\hline \multirow{8}{*}{$\begin{array}{l}\text { Polychlorinated } \\
\text { Biphenyls (PCBs) }\end{array}$} & PCB $28(\mu \mathrm{g} / \mathrm{kg}$ TS $)$ & $28 \%$ & $<0.1$ & $<0.1$ & 2.2 \\
\hline & PCB $52(\mu \mathrm{g} / \mathrm{kg}$ TS $)$ & $53 \%$ & 0.135 & $<0.1$ & 6.6 \\
\hline & PCB $101(\mu \mathrm{g} / \mathrm{kg}$ TS $)$ & $75 \%$ & 0.415 & $<0.1$ & 20 \\
\hline & PCB $118(\mu \mathrm{g} / \mathrm{kg}$ TS $)$ & $69 \%$ & 0.26 & $<0.1$ & 16 \\
\hline & PCB $153(\mu \mathrm{g} / \mathrm{kg} \mathrm{TS})$ & $75 \%$ & 0.865 & $<0.1$ & 24 \\
\hline & PCB $138(\mu \mathrm{g} / \mathrm{kg}$ TS $)$ & $75 \%$ & 0.935 & $<0.1$ & 27 \\
\hline & PCB $180(\mu \mathrm{g} / \mathrm{kg}$ TS $)$ & $72 \%$ & 0.7 & $<0.1$ & 25 \\
\hline & sum of 7 PCBs $(\mu \mathrm{g} / \mathrm{kg}$ TS $)$ & $75 \%$ & 3.2 & $<0.4$ & 100 \\
\hline \multirow{2}{*}{ Alkylphenols } & $\mathrm{OP}(\mathrm{mg} / \mathrm{kg} \mathrm{TS})$ & $38 \%$ & $<0.02$ & $<0.01$ & 0.529 \\
\hline & $\mathrm{NP}(\mathrm{mg} / \mathrm{kg} \mathrm{TS})$ & $38 \%$ & $<0.1$ & $<0.1$ & 30.5 \\
\hline \multirow{10}{*}{ Phthalates } & DMP (mg/kg TS) & $3 \%$ & $<0.05$ & $<0.05$ & 0.099 \\
\hline & DPP (mg/kg TS) & $6 \%$ & $<0.05$ & $<0.05$ & 0.7 \\
\hline & DiBP (mg/kg TS) & $19 \%$ & $<0.05$ & $<0.05$ & 0.15 \\
\hline & DBP (mg/kg TS) & $31 \%$ & $<0.05$ & $<0.05$ & 0.79 \\
\hline & DNOP (mg/kg TS) & $22 \%$ & $<0.0775$ & $<0.05$ & 7 \\
\hline & DEHP (mg/kg TS) & $66 \%$ & 1.3 & $<0.05$ & 33 \\
\hline & BBP (mg/kg TS) & $6 \%$ & $<0.05$ & $<0.05$ & 0.2 \\
\hline & DCP (mg/kg TS) & $22 \%$ & $<0.05$ & $<0.05$ & 0.84 \\
\hline & DiDP (mg/kg TS) & $28 \%$ & $<2.5$ & $<2.5$ & 22 \\
\hline & DiNP (mg/kg TS) & $31 \%$ & $<4$ & $<2.5$ & 430 \\
\hline \multirow{10}{*}{$\begin{array}{l}\text { Brominated flame } \\
\text { retardants }\end{array}$} & BDE $28(\mu \mathrm{g} / \mathrm{kg} \mathrm{TS})$ & $3 \%$ & $<0.26$ & $<0.032$ & 0.7 \\
\hline & tetraBDE $(\mu \mathrm{g} / \mathrm{kg} \mathrm{TS})$ & $9 \%$ & $<3.3$ & $<2$ & 21 \\
\hline & BDE $47(\mu \mathrm{g} / \mathrm{kg} \mathrm{TS})$ & $22 \%$ & $<0.335$ & $<0.16$ & 21 \\
\hline & pentaBDE $(\mu \mathrm{g} / \mathrm{kg}$ TS $)$ & $9 \%$ & $<4$ & $<2.4$ & 15 \\
\hline & BDE $99(\mu \mathrm{g} / \mathrm{kg} \mathrm{TS})$ & $25 \%$ & $<0.425$ & $<0.18$ & 11 \\
\hline & $\mathrm{BDE} 100(\mu \mathrm{g} / \mathrm{kg} \mathrm{TS})$ & $22 \%$ & $<0.33$ & $<0.064$ & 3.2 \\
\hline & BDE $153(\mu \mathrm{g} / \mathrm{kg}$ TS $)$ & $9 \%$ & $<0.275$ & $<0.13$ & 1.4 \\
\hline & BDE $154(\mu \mathrm{g} / \mathrm{kg} \mathrm{TS})$ & $9 \%$ & $<0.235$ & $<0.027$ & 0.6 \\
\hline & decaBDE $(\mu \mathrm{g} / \mathrm{kg} \mathrm{TS})$ & $3 \%$ & $<25.5$ & $<10$ & 110 \\
\hline & TBBP-A $(\mu \mathrm{g} / \mathrm{kg}$ TS $)$ & $9 \%$ & $<5$ & $<5$ & 6.66 \\
\hline \multirow{13}{*}{$\begin{array}{l}\text { Perfluorinated } \\
\text { substances } \\
\text { (PFASs) }\end{array}$} & PFBA $(\mu \mathrm{g} / \mathrm{kg}$ TS) & $19 \%$ & $<0.5$ & $<0.5$ & 1.42 \\
\hline & PFPeA ( $\mu \mathrm{g} / \mathrm{kg}$ TS) & $6 \%$ & $<0.5$ & $<0.5$ & 0.895 \\
\hline & PFHxA ( $\mu \mathrm{g} / \mathrm{kg}$ TS) & $3 \%$ & $<0.5$ & $<0.5$ & 0.635 \\
\hline & PFUnDA $(\mu \mathrm{g} / \mathrm{kg}$ TS) & $3 \%$ & $<0.5$ & $<0.5$ & 0.872 \\
\hline & PFDoDA $(\mu \mathrm{g} / \mathrm{kg} \mathrm{TS})$ & $6 \%$ & $<0.5$ & $<0.5$ & 1.46 \\
\hline & PFOS ( $\mu \mathrm{g} / \mathrm{kg}$ TS) & $44 \%$ & $<0.5$ & $<0.5$ & 3.18 \\
\hline & PFDS ( $\mu \mathrm{g} / \mathrm{kg}$ TS) & $9 \%$ & $<0.5$ & $<0.5$ & 0.557 \\
\hline & FOSA $(\mu \mathrm{g} / \mathrm{kg}$ TS $)$ & $9 \%$ & $<0.5$ & $<0.5$ & 0.994 \\
\hline & PFTeDA ( $\mu \mathrm{g} / \mathrm{kg}$ TS $)$ & $6 \%$ & $<0.5$ & $<0.5$ & 1.35 \\
\hline & MeFOSA $(\mu \mathrm{g} / \mathrm{kg}$ TS) & $3 \%$ & $<0.5$ & $<0.5$ & 0.584 \\
\hline & EtFOSA ( $\mu \mathrm{g} / \mathrm{kg}$ TS) & $9 \%$ & $<0.5$ & $<0.5$ & 0.735 \\
\hline & MeFOSE $(\mu \mathrm{g} / \mathrm{kg} \mathrm{TS})$ & $3 \%$ & $<0.5$ & $<0.5$ & 0.825 \\
\hline & EtFOSE $(\mu \mathrm{g} / \mathrm{kg} \mathrm{TS})$ & $13 \%$ & $<0.5$ & $<0.5$ & 1.19 \\
\hline
\end{tabular}




\begin{tabular}{|c|c|c|c|c|c|}
\hline \multirow{2}{*}{ Substance group } & \multirow{2}{*}{ Substance } & \multirow{2}{*}{$\begin{array}{c}\mathbf{f}_{\text {quant }} \\
(\%)\end{array}$} & \multicolumn{3}{|c|}{ Concentrations } \\
\hline & & & Median & Minimum & Maximum \\
\hline \multirow{7}{*}{ Organotins } & MBT $(\mu \mathrm{g} / \mathrm{kg} \mathrm{TS})$ & $66 \%$ & 12 & $<1$ & 231 \\
\hline & DBT $(\mu \mathrm{g} / \mathrm{kg}$ TS $)$ & $69 \%$ & 12 & $<1$ & 781 \\
\hline & TBT $(\mu \mathrm{g} / \mathrm{kg}$ TS $)$ & $44 \%$ & $<1$ & $<1$ & 31.3 \\
\hline & MOT $(\mu \mathrm{g} / \mathrm{kg}$ TS $)$ & $47 \%$ & $<1$ & $<1$ & 23.4 \\
\hline & DOT $(\mu \mathrm{g} / \mathrm{kg} \mathrm{TS})$ & $47 \%$ & $<1$ & $<1$ & 40.7 \\
\hline & MPhT $(\mu \mathrm{g} / \mathrm{kg} \mathrm{TS})$ & $13 \%$ & $<1$ & $<1$ & 10.1 \\
\hline & DPhT $(\mu \mathrm{g} / \mathrm{kg}$ TS $)$ & $6 \%$ & $<1$ & $<1$ & 2.69 \\
\hline \multirow{11}{*}{ Pesticides } & o,p'-DDT (mg/kg TS) & $3 \%$ & $<0.01$ & $<0.01$ & 0.25 \\
\hline & p,p'-DDT (mg/kg TS) & $6 \%$ & $<0.01$ & $<0.01$ & 1.58 \\
\hline & o,p'-DDD (mg/kg TS) & $3 \%$ & $<0.01$ & $<0.01$ & 0.328 \\
\hline & p,p'-DDD (mg/kg TS) & $3 \%$ & $<0.01$ & $<0.01$ & 0.869 \\
\hline & o,p'-DDE (mg/kg TS) & $3 \%$ & $<0.01$ & $<0.01$ & 0.02 \\
\hline & p,p'-DDE (mg/kg TS) & $3 \%$ & $<0.01$ & $<0.01$ & 0.216 \\
\hline & Terbuthylazine-desethyl-2-hydroxy (mg/kg TS) & $3 \%$ & $<0.01$ & $<0.01$ & 0.1 \\
\hline & Hydroxy-terbutylazine (mg/kg TS) & $3 \%$ & $<0.01$ & $<0.01$ & 0.1 \\
\hline & Carbendazim (mg/kg TS) & $3 \%$ & $<0.01$ & $<0.01$ & 0.1 \\
\hline & Propiconazole (mg/kg TS) & $6 \%$ & $<0.01$ & $<0.01$ & 0.1 \\
\hline & Terbutryn (mg/kg TS) & $3 \%$ & $<0.01$ & $<0.01$ & 0.1 \\
\hline
\end{tabular}

Table S3: Frequency of quantification $\left(f_{\text {quant }}\right)$, minimal, maximal and median concentrations for compounds quantified in at least one sample 
IV. Correlation test results between quantified concentrations of each substance and the total number of substances quantified in each sample

\begin{tabular}{|c|c|c|c|}
\hline Substance & $\mathbf{f}_{\text {censored }}$ & P-value & tau \\
\hline $\mathrm{C}_{10}-\mathrm{C}_{12}$ Aliphatics* & 0.59 & 4.25E-07 & 0.542 \\
\hline $\mathrm{C}_{12}-\mathrm{C}_{16}$ Aliphatics* & 0.47 & 2.07E-08 & 0.649 \\
\hline $\mathrm{C}_{16}-\mathrm{C}_{35}$ Aliphatics* & 0.03 & $5.16 \mathrm{E}-09$ & 0.726 \\
\hline $\mathrm{C}_{10}-\mathrm{C}_{16}$ Aromatics & 0.72 & 0.963 & 0.006 \\
\hline $\mathrm{C}_{16}-\mathrm{C}_{35}$ Aromatics* & 0.75 & $2.42 \mathrm{E}-05$ & 0.373 \\
\hline Phen* & 0.63 & $6.18 \mathrm{E}-07$ & 0.520 \\
\hline Fluo* & 0.50 & $1.01 \mathrm{E}-08$ & 0.653 \\
\hline Pyr* & 0.47 & $2.6 \mathrm{E}-09$ & 0.690 \\
\hline $\mathrm{BaA}^{*}$ & 0.63 & $2.22 \mathrm{E}-07$ & 0.540 \\
\hline Chry* & 0.59 & $2.86 \mathrm{E}-07$ & 0.550 \\
\hline $\mathrm{BbF}^{*}$ & 0.50 & $1.25 \mathrm{E}-08$ & 0.649 \\
\hline $\mathrm{BkF}^{*}$ & 0.75 & $4.02 \mathrm{E}-05$ & 0.363 \\
\hline $\mathrm{BaP}^{*}$ & 0.66 & 4.01E-07 & 0.512 \\
\hline BPer* & 0.56 & 2.63E-08 & 0.611 \\
\hline IP* & 0.69 & $5.76 \mathrm{E}-06$ & 0.442 \\
\hline Formaldehyde* & 0.03 & 0.00175 & 0.389 \\
\hline Acetaldehyde* & 0.13 & 0.00398 & 0.357 \\
\hline BDE 99* & 0.75 & 0.00278 & 0.359 \\
\hline PCB28* & 0.72 & 0.00062 & 0.369 \\
\hline PCB52* & 0.47 & $3.18 \mathrm{E}-09$ & 0.685 \\
\hline PCB101* & 0.25 & $5.37 \mathrm{E}-10$ & 0.762 \\
\hline PCB118* & 0.31 & 7.17E-09 & 0.704 \\
\hline PCB153* & 0.25 & $7 \mathrm{E}-11$ & 0.800 \\
\hline PCB138* & 0.25 & 5.62E-11 & 0.804 \\
\hline PCB180* & 0.28 & $9.11 \mathrm{E}-11$ & 0.792 \\
\hline sum of 7 PCBs* & 0.25 & $4.55 \mathrm{E}-11$ & 0.808 \\
\hline PFOS* & 0.56 & $5.9 \mathrm{E}-05$ & 0.442 \\
\hline $\mathrm{OP}^{*}$ & 0.63 & $1.49 \mathrm{E}-05$ & 0.500 \\
\hline $\mathrm{NP}^{*}$ & 0.63 & 3.87E-07 & 0.542 \\
\hline DBP* & 0.69 & 0.00017 & 0.389 \\
\hline DEHP* & 0.34 & 4.19E-09 & 0.710 \\
\hline DiDP* & 0.72 & 0.00681 & 0.300 \\
\hline DiNP* & 0.69 & 0.00406 & 0.329 \\
\hline Monobutyltin* & 0.34 & 7.67E-09 & 0.698 \\
\hline Dibutyltin* & 0.31 & $2.16 \mathrm{E}-09$ & 0.728 \\
\hline Tributyltin* & 0.56 & 0.000127 & 0.421 \\
\hline Monooctyltin* & 0.53 & 2.94E-06 & 0.524 \\
\hline Dioctyltin* & 0.53 & $1.23 \mathrm{E}-07$ & 0.593 \\
\hline
\end{tabular}

Table S4: Results of the Kendall's tau test between censored substance concentrations, $n=32$. Significant correlations, considering $\alpha=0.01$ are marked with a $*$ The test was only computed for substances quantified in at least 8 of the 32 analyzed samples ( $f_{\text {cens }} \leq 75 \%$ censored). 


\section{Statistics addressing inter-site variability}

\begin{tabular}{|l|c|c|}
\hline Substance & fcensored & $\begin{array}{c}\text { Inter-site } \\
\text { factor of } \\
\text { variation }\end{array}$ \\
\hline $\mathrm{C}_{10}-\mathrm{C}_{12}$ Aliphatics & 0.60 & 14.6 \\
\hline $\mathrm{C}_{12} \mathrm{C}_{16}$ Aliphatics & 0.50 & 28.5 \\
\hline $\mathrm{C}_{16} \mathrm{C}_{35}$ Aliphatics & 0.03 & 382 \\
\hline $\mathrm{C}_{10}-\mathrm{C}_{16}$ Aromatics & 0.73 & 14.6 \\
\hline Phen & 0.63 & 7.9 \\
\hline Fluo & 0.50 & 15.0 \\
\hline Pyr & 0.50 & 17.6 \\
\hline BaA & 0.63 & 5.8 \\
\hline Chry & 0.60 & 4.9 \\
\hline BbF & 0.53 & 5.6 \\
\hline BaP & 0.67 & 5.6 \\
\hline BPer & 0.57 & 6.9 \\
\hline IP & 0.70 & 4.1 \\
\hline Formaldehyde & 0.03 & 22.1 \\
\hline Acetaldehyde & 0.13 & 16.4 \\
\hline PCB28 & 0.73 & 22.0 \\
\hline PCB52 & 0.47 & 66.0 \\
\hline PCB101 & 0.27 & 200 \\
\hline PCB118 & 0.30 & 160 \\
\hline PCB153 & 0.27 & 240 \\
\hline PCB138 & 0.27 & 270 \\
\hline PCB180 & 0.30 & 250 \\
\hline PFOS & 0.60 & 6.4 \\
\hline OP & 0.63 & 52.9 \\
\hline NP & 0.63 & 305 \\
\hline DBP & 0.70 & 15.8 \\
\hline DEHP & 0.37 & 660 \\
\hline DiDP & 0.70 & 40.0 \\
\hline DiNP & 0.67 & 172 \\
\hline Monobutyltin & 0.37 & 231 \\
\hline Dibutyltin & 0.33 & 781 \\
\hline Tributyltin & 0.60 & 31.3 \\
\hline Monooctyltin & 23.4 \\
\hline Dioctyltin & 40.7 \\
\hline & \\
\hline
\end{tabular}

Table S5: Summary of factors of variability within ponds for substances quantified in at least $25 \%$ of samples 


\begin{tabular}{|l|c|c|c|c|c|}
\hline \multicolumn{1}{|c|}{ Substance } & f $_{\text {censored }}$ & $\begin{array}{c}\text { P-value } \\
\text { (all) }\end{array}$ & $\begin{array}{c}\text { P-value } \\
\text { (IC-Res) }\end{array}$ & $\begin{array}{c}\text { P-value } \\
\text { (IC-Ro) }\end{array}$ & $\begin{array}{c}\text { P-value } \\
\text { (Res-Ro) }\end{array}$ \\
\hline C $10_{10}$ C $12_{2}$ Aliphatics & 0.60 & 0.030 & $\mathbf{0 . 0 0 1 3}$ & 0.91 & $\mathbf{0 . 0 0 1 3}$ \\
\hline${\text { C } 12-C_{16} \text { Aliphatics }}$ & 0.50 & 0.12 & $\mathbf{0 . 0 0 7 1}$ & 0.83 & $\mathbf{0 . 0 0 7 1}$ \\
\hline C $_{16}$ C $_{35}$ Aliphatics & 0.03 & 0.042 & $\mathbf{0 . 0 0 0 8 0}$ & 0.60 & $\mathbf{0 . 0 0 0 8 2}$ \\
\hline${\text { C } 10-C_{16} \text { Aromatics }}$ & 0.73 & 0.018 & 1 & $\mathbf{0 . 0 0 4 7}$ & 1 \\
\hline Phen & 0.63 & 0.011 & $\mathbf{0 . 0 0 1 3}$ & 0.18 & $\mathbf{0 . 0 0 1 3}$ \\
\hline Fluo & 0.50 & 0.041 & $\mathbf{0 . 0 0 0 8 2}$ & 0.38 & $\mathbf{0 . 0 0 0 8 2}$ \\
\hline Pyr & 0.50 & 0.041 & $\mathbf{0 . 0 0 0 3 2}$ & 0.35 & $\mathbf{0 . 0 0 0 3 2}$ \\
\hline BaA & 0.63 & 0.14 & 0.045 & 0.75 & 0.045 \\
\hline Chry & 0.60 & 0.22 & 0.054 & 0.69 & 0.054 \\
\hline BbF & 0.53 & 0.15 & 0.013 & 0.59 & 0.013 \\
\hline BaP & 0.67 & 0.12 & 0.58 & 0.46 & 0.578515 \\
\hline BPer & 0.57 & 0.049 & $\mathbf{0 . 0 0 2 6}$ & 0.67 & $\mathbf{0 . 0 0 2 6}$ \\
\hline IP & 0.70 & 0.082 & 0.046 & 0.75 & 0.046 \\
\hline Formaldehyde & 0.03 & 0.42 & 0.19 & 0.32 & 0.19 \\
\hline Acetaldehyde & 0.13 & 0.48 & 0.80 & 0.48 & 0.80 \\
\hline PCB28 & 0.73 & 0.048 & 0.011 & 0.10 & 0.011 \\
\hline PCB52 & 0.47 & 0.054 & $\mathbf{0 . 0 0 7 1}$ & 0.19 & $\mathbf{0 . 0 0 7 1}$ \\
\hline PCB101 & 0.27 & 0.15 & 0.012 & 0.31 & 0.012 \\
\hline PCB118 & 0.30 & 0.12 & $\mathbf{0 . 0 0 3 8}$ & 0.31 & $\mathbf{0 . 0 0 3 8}$ \\
\hline PCB153 & 0.27 & 0.21 & 0.012 & 0.52 & 0.012 \\
\hline PCB138 & 0.27 & 0.29 & 0.026 & 0.63 & 0.026 \\
\hline PCB180 & 0.30 & 0.25 & 0.033 & 0.60 & 0.033 \\
\hline PFOS & 0.60 & 0.48 & 0.19 & 0.63 & 0.19 \\
\hline OP & 0.63 & $\mathbf{0 . 0 0 2 2}$ & $\mathbf{0 . 0 0 9 4}$ & 0.04 & $\mathbf{0 . 0 0 9 4}$ \\
\hline NP & 0.63 & 0.027 & 0.046 & 0.39 & 0.046 \\
\hline DBP & 0.70 & 0.27 & 0.12 & 0.22 & 0.12 \\
\hline DEHP & 0.37 & 0.11 & $\mathbf{0 . 0 0 6 9}$ & 0.35 & $\mathbf{0 . 0 0 6 9}$ \\
\hline DiDP & 0.70 & 0.37 & $\mathbf{0 . 0 0 2 7}$ & 0.76 & $\mathbf{0 . 0 0 2 7}$ \\
\hline DiNP & 0.67 & 0.037 & $\mathbf{0 . 0 0 7 6}$ & 0.12 & $\mathbf{0 . 0 0 7 6}$ \\
\hline Monobutyltin & 0.37 & 0.013 & $\mathbf{0 . 0 0 1 8}$ & 0.10 & $\mathbf{0 . 0 0 1 8}$ \\
\hline Dibutyltin & 0.33 & 0.081 & $\mathbf{0 . 0 0 3 5}$ & 0.36 & $\mathbf{0 . 0 0 3 5}$ \\
\hline Tributyltin & 0.60 & 0.18 & 0.054 & 0.63 & 0.053592 \\
\hline Monooctyltin & 0.57 & 0.039 & $\mathbf{0 . 0 0 4 5}$ & 0.24 & $\mathbf{0 . 0 0 4 5}$ \\
\hline Dioctyltin & 0.040 & $\mathbf{0 . 0 0 2 6}$ & 0.76 & $\mathbf{0 . 0 0 2 6}$ \\
\hline
\end{tabular}

Table S6: Results of the Peto\&Peto generalized Wilcoxon test for differences between substance concentrations observed in each city, $\mathrm{n}=32$, $\mathrm{n}_{\mathrm{IC}}=18, \mathrm{n}_{\mathrm{Res}}=9, \mathrm{n}_{\mathrm{R}}=5$. P-values for significant differences, considering $\alpha=0.01$, are in bold. The tests were only computed for substances for which $\mathrm{f}_{\text {censored }}<75 \%$. 


\begin{tabular}{|c|c|c|c|c|c|c|}
\hline Substance & $\mathbf{f}_{\text {censored }}$ & $\begin{array}{c}\text { P-value } \\
\text { (Catchment } \\
\text { area) }\end{array}$ & $\begin{array}{c}\text { P-value } \\
\text { (Ratio facility } \\
\text { to catchment) }\end{array}$ & $\begin{array}{c}\text { P-value } \\
\text { (Facility } \\
\text { age) }\end{array}$ & $\begin{array}{c}\text { P-value } \\
\text { (Sediment } \\
\text { age) }\end{array}$ & $\begin{array}{c}\text { P-value } \\
\text { (Imperviousness) }\end{array}$ \\
\hline $\mathrm{C}_{10}-\mathrm{C}_{12}$ Aliphatics & 0.60 & 0.54 & 0.44 & 0.068 & 0.068 & 0.077 \\
\hline $\mathrm{C}_{12}-\mathrm{C}_{16}$ Aliphatics & 0.50 & 0.70 & 0.36 & 0.11 & 0.25 & 0.16 \\
\hline $\mathrm{C}_{16}-\mathrm{C}_{35}$ Aliphatics & 0.03 & 1 & 0.41 & 0.011 & 0.044 & 0.073 \\
\hline $\mathrm{C}_{10}-\mathrm{C}_{16}$ Aromatics & 0.73 & 0.81 & 0.73 & 0.84 & 0.84 & 0.86 \\
\hline Phen & 0.63 & 0.22 & 0.85 & 0.13 & 0.22 & 0.19 \\
\hline Fluo & 0.50 & 0.62 & 0.41 & 0.18 & 0.097 & 0.14 \\
\hline Pyr & 0.50 & 0.64 & 0.46 & 0.24 & 0.085 & 0.23 \\
\hline $\mathrm{BaA}$ & 0.63 & 0.73 & 0.49 & 0.21 & 0.063 & 0.25 \\
\hline Chry & 0.60 & 0.93 & 0.34 & 0.31 & 0.071 & 0.22 \\
\hline $\mathrm{BbF}$ & 0.53 & 0.83 & 0.40 & 0.22 & 0.057 & 0.20 \\
\hline $\mathrm{BaP}$ & 0.67 & 0.81 & 0.38 & 0.27 & 0.0078 & 0.11 \\
\hline BPer & 0.57 & 0.58 & 0.61 & 0.11 & 0.043 & 0.11 \\
\hline IP & 0.70 & 0.94 & 0.59 & 0.096 & 0.0053 & 0.28 \\
\hline Formaldehyde & 0.03 & 0.86 & 0.50 & 0.042 & 0.021 & 0.35 \\
\hline Acetaldehyde & 0.13 & 0.76 & 0.79 & 0.081 & 0.0013 & 0.25 \\
\hline PCB28 & 0.73 & 0.84 & 0.51 & 0.65 & 0.53 & 0.59 \\
\hline PCB52 & 0.47 & 0.71 & 0.25 & 0.19 & 0.32 & 0.034 \\
\hline PCB101 & 0.27 & 0.51 & 0.05 & 0.058 & 0.58 & 0.33 \\
\hline PCB118 & 0.30 & 0.66 & 0.14 & 0.077 & 0.32 & 0.39 \\
\hline PCB153 & 0.27 & 0.51 & 0.05 & 0.045 & 0.49 & 0.25 \\
\hline PCB138 & 0.27 & 0.36 & 0.03 & 0.063 & 0.54 & 0.28 \\
\hline PCB180 & 0.30 & 0.36 & 0.04 & 0.044 & 0.38 & 0.21 \\
\hline PFOS & 0.60 & 0.66 & 0.10 & 0.36 & 0.81 & 0.44 \\
\hline $\mathrm{OP}$ & 0.63 & 0.18 & 0.98 & 0.22 & 0.25 & 0.077 \\
\hline $\mathrm{NP}$ & 0.63 & 1 & 0.41 & 0.078 & 0.012 & 0.087 \\
\hline DBP & 0.70 & 0.87 & 0.29 & 0.50 & 0.80 & 0.57 \\
\hline DEHP & 0.37 & 0.87 & 0.07 & 0.077 & 0.75 & 0.12 \\
\hline DiDP & 0.70 & 0.64 & 0.42 & 0.041 & 0.22 & 0.039 \\
\hline DiNP & 0.67 & 0.84 & 0.61 & 0.10 & 0.12 & 0.045 \\
\hline Monobutyltin & 0.37 & 0.45 & 0.64 & 0.027 & 0.12 & 0.13 \\
\hline Dibutyltin & 0.33 & 0.75 & 0.30 & 0.014 & 0.11 & 0.21 \\
\hline Tributyltin & 0.60 & 0.38 & 1 & 0.034 & 0.22 & 1 \\
\hline Monooctyltin & 0.57 & 0.27 & 0.89 & 0.022 & 0.027 & 0.13 \\
\hline Dioctyltin & 0.57 & 0.50 & 0.87 & 0.12 & 0.072 & 0.064 \\
\hline
\end{tabular}

Table S7: Results of Kendall's tau test for correlation between censored concentrations and catchment and facility properties, $\mathrm{n}=30$ for all except imperviousness for which $\mathrm{n}=21 \mathrm{P}$-values for significant correlations, considering $\alpha=0.01$, are in bold. The test was only computed for substances for which $\mathrm{f}_{\text {censored }}<75 \%$. 


\begin{tabular}{|c|c|c|c|c|c|c|c|c|}
\hline Substance & $\mathbf{f}_{\text {censored }}$ & $\begin{array}{c}\text { P-value } \\
\text { (all) }\end{array}$ & $\begin{array}{l}\text { P-value } \\
\text { (Or-Os) }\end{array}$ & $\begin{array}{l}\text { P-value } \\
\text { (Or-St) }\end{array}$ & $\begin{array}{l}\text { P-value } \\
\text { (Or-Va) }\end{array}$ & $\begin{array}{l}\text { P-value } \\
\text { (Os-St) }\end{array}$ & $\begin{array}{l}\text { P-value } \\
\text { (Os-Va) }\end{array}$ & $\begin{array}{l}\text { P-value } \\
\text { (St-Va) }\end{array}$ \\
\hline $\mathrm{C}_{10}-\mathrm{C}_{12}$ Aliphatics & 0.60 & 0.0027 & $6.3 \mathrm{E}-05$ & 0.0036 & 0.00053 & 0.17 & 0.77 & 0.42 \\
\hline $\mathrm{C}_{12}-\mathrm{C}_{16}$ Aliphatics & 0.50 & 0.00030 & 6.3E-05 & 0.0013 & 0.00021 & 0.092 & 0.35 & 0.31 \\
\hline $\mathrm{C}_{16}-\mathrm{C}_{35}$ Aliphatics & 0.03 & $1.6 \mathrm{E}-06$ & $6.3 \mathrm{E}-05$ & $2.7 \mathrm{E}-06$ & 4.6E-06 & 0.041 & 0.77 & 0.062 \\
\hline $\mathrm{C}_{10}-\mathrm{C}_{16}$ Aromatics & 0.73 & 0.90 & 1 & 1 & 1 & 0.58 & 0.65 & 0.48 \\
\hline Phen & 0.63 & 0.018 & 0.014 & 0.0036 & 0.00053 & 0.77 & 1 & 0.89 \\
\hline Fluo & 0.50 & 0.0014 & $6.3 \mathrm{E}-05$ & $8.6 \mathrm{E}-05$ & 0.00053 & 0.92 & 0.42 & 0.75 \\
\hline Pyr & 0.50 & 0.00056 & $6.3 \mathrm{E}-05$ & $8.6 \mathrm{E}-05$ & $6.3 \mathrm{E}-05$ & 0.69 & 0.42 & 0.68 \\
\hline $\mathrm{BaA}$ & 0.63 & 0.0095 & 0.014 & 0.0036 & 0.00053 & 0.77 & 0.28 & 0.54 \\
\hline Chry & 0.60 & 0.0044 & 0.014 & 0.0012 & 0.00053 & 0.46 & 0.28 & 0.48 \\
\hline $\mathrm{BbF}$ & 0.53 & 0.00059 & $6.3 \mathrm{E}-05$ & 0.00035 & $6.3 \mathrm{E}-05$ & 0.77 & 0.42 & 0.31 \\
\hline $\mathrm{BaP}$ & 0.67 & 0.0083 & $6.3 \mathrm{E}-05$ & 0.025 & 0.00053 & 0.28 & 0.42 & 0.36 \\
\hline BPer & 0.57 & 0.0027 & $6.3 \mathrm{E}-05$ & 0.0012 & 0.00053 & 0.40 & 0.42 & 0.37 \\
\hline IP & 0.70 & 0.0054 & 0.014 & 0.025 & 0.00053 & 0.91 & 0.28 & 0.12 \\
\hline Formaldehyde & 0.03 & 0.00024 & 0.35 & 0.0040 & $4.5 \mathrm{E}-05$ & 0.91 & 1 & 0.011 \\
\hline Acetaldehyde & 0.13 & \begin{tabular}{|l}
0.000018 \\
\end{tabular} & 0.063 & 0.24 & $2.3 \mathrm{E}-05$ & 0.46 & 0.88 & 0.00040 \\
\hline PCB28 & 0.73 & 0.014 & 1 & 0.0012 & 0.055 & 0.35 & 0.43 & 0.57 \\
\hline PCB52 & 0.47 & 0.00062 & $6.3 \mathrm{E}-05$ & $1.7 \mathrm{E}-05$ & 0.00053 & 0.85 & 0.89 & 0.79 \\
\hline PCB101 & 0.27 & 0.000087 & $6.3 \mathrm{E}-05$ & $2.7 \mathrm{E}-06$ & 0.00010 & 0.85 & 0.42 & 0.55 \\
\hline PCB118 & 0.30 & \begin{tabular}{|l|}
0.00037 \\
\end{tabular} & $6.3 \mathrm{E}-05$ & $6.0 \mathrm{E}-06$ & 0.0013 & 0.85 & 0.59 & 0.43 \\
\hline PCB153 & 0.27 & 0.000026 & $6.3 \mathrm{E}-05$ & $3.0 \mathrm{E}-06$ & $8.1 \mathrm{E}-06$ & 0.54 & 0.42 & 0.40 \\
\hline PCB138 & 0.27 & \begin{tabular}{|l|l|}
0.000025 \\
\end{tabular} & $6.3 \mathrm{E}-05$ & $4.0 \mathrm{E}-06$ & $5.3 \mathrm{E}-06$ & 0.54 & 0.59 & 0.38 \\
\hline PCB180 & 0.30 & 0.000015 & $6.3 \mathrm{E}-05$ & $2.7 \mathrm{E}-06$ & 4.6E-06 & 0.28 & 0.78 & 0.28 \\
\hline PFOS & 0.60 & 0.0052 & 0.014 & 0.0012 & $6.3 \mathrm{E}-05$ & 1 & 0.88 & 0.82 \\
\hline $\mathrm{OP}$ & 0.63 & 0.0099 & $6.3 \mathrm{E}-05$ & 0.0098 & 0.00053 & 0.88 & 0.53 & 0.59 \\
\hline $\mathrm{NP}$ & 0.63 & 0.0011 & $6.3 \mathrm{E}-05$ & 0.025 & $6.3 \mathrm{E}-05$ & 0.17 & 0.77 & 0.15 \\
\hline DBP & 0.70 & 0.0093 & 1 & 0.0012 & 0.0082 & 0.19 & 0.27 & 0.77 \\
\hline DEHP & 0.37 & $6.2 \mathrm{E}-05$ & $6.3 \mathrm{E}-05$ & $7.2 \mathrm{E}-06$ & $4.6 \mathrm{E}-06$ & 0.85 & 0.89 & 0.75 \\
\hline DiDP & 0.70 & 0.0023 & $6.3 \mathrm{E}-05$ & 0.0027 & 0.0018 & 0.18 & 0.92 & 0.32 \\
\hline DiNP & 0.67 & 0.0027 & $6.3 \mathrm{E}-05$ & 0.0024 & 0.0031 & 0.18 & 0.81 & 0.44 \\
\hline Monobutyltin & 0.37 & \begin{tabular}{|l|}
$7.7 \mathrm{E}-05$ \\
\end{tabular} & $6.3 \mathrm{E}-05$ & $3.1 \mathrm{E}-05$ & 4.6E-06 & 1 & 0.42 & 0.44 \\
\hline Dibutyltin & 0.33 & 7.2E-06 & $6.3 \mathrm{E}-05$ & $4.5 \mathrm{E}-06$ & 4.6E-06 & 0.85 & 0.42 & 0.093 \\
\hline Tributyltin & 0.60 & 0.0011 & 0.46 & 0.065 & 0.00056 & 0.69 & 0.14 & 0.024 \\
\hline Monooctyltin & 0.57 & 0.00026 & 6.3E-05 & 0.0036 & $4.6 \mathrm{E}-06$ & 0.27 & 0.55 & 0.17 \\
\hline Dioctyltin & 0.57 & 3.3E-11 & $6.3 \mathrm{E}-05$ & 0.0036 & 4.6E-06 & 0.00013 & 0.0023 & 0.49 \\
\hline
\end{tabular}

Table S8: Results of the Peto\&Peto generalized Wilcoxon test for differences between substance concentrations observed in each city, $\mathrm{n}=32$, $\mathrm{n}_{\mathrm{s}}=2, \mathrm{nOr}_{\mathrm{r}}=12, \mathrm{n}_{\mathrm{St}}=11, \mathrm{n}_{\mathrm{Va}}=7$. P-values for significant differences, considering $\alpha=0.01$, are in bold. The tests were only computed for substances for which $\mathrm{f}_{\text {censored }}<75 \%$. 
VI. Statistics comparing inlet and outlet concentrations

\begin{tabular}{|c|c|c|c|}
\hline Substance & $\mathbf{f}_{\text {censored }}$ & P-value & tau \\
\hline $\mathrm{C}_{10}-\mathrm{C}_{12}$ Aliphatics* & 0.60 & 0.0052 & 0.381 \\
\hline $\mathrm{C}_{12}-\mathrm{C}_{16}$ Aliphatics* & 0.50 & 0.0097 & 0.419 \\
\hline $\mathrm{C}_{16}-\mathrm{C}_{35}$ Aliphatics* & 0.03 & 0.00063 & 0.667 \\
\hline $\mathrm{C}_{10}-\mathrm{C}_{16}$ Aromatics & 0.73 & 1 & -0.010 \\
\hline Phen & 0.63 & 0.018 & 0.305 \\
\hline Fluo* & 0.50 & 0.00093 & 0.533 \\
\hline Pyr* & 0.50 & 0.00076 & 0.543 \\
\hline $\mathrm{BaA}$ & 0.63 & 0.016 & 0.295 \\
\hline Chry & 0.60 & 0.015 & 0.333 \\
\hline $\mathrm{BbF}$ & 0.53 & 0.011 & 0.390 \\
\hline $\mathrm{BaP}^{*}$ & 0.67 & 0.00023 & 0.419 \\
\hline BPer* & 0.57 & 0.0032 & 0.419 \\
\hline IP & 0.70 & 0.015 & 0.257 \\
\hline Formaldehyde & 0.03 & 0.164 & 0.276 \\
\hline Acetaldehyde & 0.13 & 0.0198 & 0.448 \\
\hline PCB28 & 0.73 & 0.047 & 0.276 \\
\hline PCB52* & 0.47 & 0.0021 & 0.514 \\
\hline PCB101* & 0.27 & 0.00025 & 0.695 \\
\hline PCB118* & 0.30 & 0.00066 & 0.638 \\
\hline PCB153* & 0.27 & $4.6 \mathrm{E}-05$ & 0.771 \\
\hline PCB138* & 0.27 & $5.6 \mathrm{E}-05$ & 0.762 \\
\hline PCB180* & 0.30 & 0.00020 & 0.695 \\
\hline PFOS* & 0.60 & 0.0061 & 0.448 \\
\hline OP* & 0.63 & 0.0014 & 0.438 \\
\hline $\mathrm{NP}$ & 0.63 & 0.014 & 0.305 \\
\hline DBP* & 0.70 & 0.00023 & 0.667 \\
\hline DEHP & 0.37 & 0.042 & 0.305 \\
\hline DiDP & 0.70 & 0.043 & 0.314 \\
\hline DiNP* & 0.67 & 0.00035 & 0.648 \\
\hline Monobutyltin* & 0.37 & 0.00055 & 0.638 \\
\hline Dibutyltin* & 0.33 & 0.0065 & 0.371 \\
\hline Tributyltin* & 0.60 & 0.0098 & 0.381 \\
\hline Monooctyltin* & 0.57 & $1.2 \mathrm{E}-05$ & 0.638 \\
\hline Dioctyltin* & 0.57 & 0.0061 & 0.448 \\
\hline
\end{tabular}

Table S9: Results of the Kendall's tau test for correlation between censored inlet and outlet substance concentrations, $n=15$ paired inlet-outlet concentrations. Significant correlations considering $\alpha=0.01$ are marked with a $*$. The test was only computed for substances for which 


\begin{tabular}{|l|c|c|c|c|}
\hline \multicolumn{1}{|c|}{ Substance } & fcensored & $\begin{array}{c}\text { Median factor } \\
\text { of variation } \\
\text { between inlet- } \\
\text { outlet samples }\end{array}$ & $\begin{array}{c}\text { Minimum } \\
\text { factor of } \\
\text { variation } \\
\text { between inlet- } \\
\text { outlet samples }\end{array}$ & $\begin{array}{c}\text { Maximum } \\
\text { factor of } \\
\text { variation } \\
\text { between inlet- } \\
\text { outlet samples }\end{array}$ \\
\hline $\mathrm{C}_{10}-\mathrm{C}_{12}$ Aliphatics & 0.60 & 1.0 & 1.0 & 14.6 \\
\hline $\mathrm{C}_{12} \mathrm{C}_{16}$ Aliphatics & 0.50 & 1.0 & 1.2 & 21.7 \\
\hline $\mathrm{C}_{16} \mathrm{C}_{35}$ Aliphatics & 0.03 & 1.1 & 1.4 & 17.4 \\
\hline $\mathrm{C}_{10} \mathrm{C}_{16}$ Aromatics & 0.73 & 1.0 & 1.0 & 5.7 \\
\hline Phen & 0.63 & 1.0 & 1.0 & 4.3 \\
\hline Fluo & 0.50 & 1.0 & 1.1 & 8.1 \\
\hline Pyr & 0.50 & 1.0 & 1.0 & 11.5 \\
\hline BaA & 0.63 & 1.0 & 1.0 & 3.1 \\
\hline Chry & 0.60 & 1.0 & 1.1 & 2.8 \\
\hline BbF & 0.53 & 1.0 & 1.1 & 7.7 \\
\hline BaP & 0.67 & 1.0 & 1.0 & 3.9 \\
\hline BPer & 0.57 & 1.0 & 1.1 & 6.2 \\
\hline IP & 0.70 & 1.0 & 1.0 & 4.1 \\
\hline Formaldehyde & 0.03 & 1.0 & 2.0 & 14.5 \\
\hline Acetaldehyde & 0.13 & 1.0 & 1.5 & 5.2 \\
\hline PCB28 & 0.73 & 1.0 & 1.0 & 10.0 \\
\hline PCB52 & 0.47 & 1.0 & 1.1 & 9.6 \\
\hline PCB101 & 0.27 & 1.0 & 1.3 & 21.9 \\
\hline PCB118 & 0.30 & 1.0 & 1.1 & 17.0 \\
\hline PCB153 & 0.27 & 1.0 & 1.2 & 21.7 \\
\hline PCB138 & 0.27 & 1.0 & 1.2 & 22.4 \\
\hline PCB180 & 0.30 & 1.0 & 1.1 & 25.5 \\
\hline PFOS & 0.60 & 1.0 & 1.0 & 4.1 \\
\hline OP & 0.63 & 1.0 & 1.0 & 11.9 \\
\hline NP & 0.63 & 1.0 & 1.0 & 59.7 \\
\hline DBP & 0.70 & 1.0 & 1.0 & 4.0 \\
\hline DEHP & 0.37 & 1.0 & 1.3 & 27.9 \\
\hline DiDP & 0.70 & 1.0 & 1.1 & 6.0 \\
\hline DiNP & 0.67 & 1.0 & 1.5 & 39.1 \\
\hline Monobutyltin & 1.0 & 1.7 & 15.1 \\
\hline Dibutyltin & 1.0 & 1.8 & 35.0 \\
\hline Tributyltin & 1.0 & 1.1 & 13.4 \\
\hline Monooctyltin & & 1.1 & 15.5 \\
\hline Dioctyltin & 0.0 & 1.0 & 3.9 \\
\hline
\end{tabular}

Table S10: Summary of factors of variability within ponds for substances quantified in at least $25 \%$ of samples 


\begin{tabular}{|c|c|c|}
\hline Substance & $\mathbf{f}_{\text {censored }}$ & P-value \\
\hline $\mathrm{C}_{10}-\mathrm{C}_{12}$ Aliphatics & 0.60 & 0.390 \\
\hline $\mathrm{C}_{12}-\mathrm{C}_{16}$ Aliphatics & 0.50 & 0.990 \\
\hline $\mathrm{C}_{16}-\mathrm{C}_{35}$ Aliphatics & 0.03 & 0.858 \\
\hline $\mathrm{C}_{10}-\mathrm{C}_{16}$ Aromatics & 0.73 & 0.410 \\
\hline Phen & 0.63 & 0.569 \\
\hline Fluo & 0.50 & 0.638 \\
\hline Pyr & 0.50 & 0.661 \\
\hline $\mathrm{BaA}$ & 0.63 & 0.293 \\
\hline Chry & 0.60 & 0.461 \\
\hline $\mathrm{BbF}$ & 0.53 & 0.506 \\
\hline $\mathrm{BaP}$ & 0.67 & 0.456 \\
\hline BPer & 0.57 & 0.289 \\
\hline IP & 0.70 & 0.661 \\
\hline Formaldehyde & 0.03 & 0.749 \\
\hline Acetaldehyde & 0.13 & 0.608 \\
\hline PCB28 & 0.73 & 0.903 \\
\hline PCB52 & 0.47 & 0.813 \\
\hline PCB101 & 0.27 & 0.981 \\
\hline PCB118 & 0.30 & 0.780 \\
\hline PCB153 & 0.27 & 0.875 \\
\hline PCB138 & 0.27 & 0.931 \\
\hline PCB180 & 0.30 & 0.795 \\
\hline PFOS & 0.60 & 0.322 \\
\hline OP & 0.63 & 0.743 \\
\hline NP & 0.63 & 0.842 \\
\hline DBP & 0.70 & 0.767 \\
\hline DEHP & 0.37 & 0.599 \\
\hline DiDP & 0.70 & 0.756 \\
\hline DiNP & 0.67 & 0.544 \\
\hline Monobutyltin & 0.37 & 0.811 \\
\hline Dibutyltin & 0.33 & 0.892 \\
\hline Tributyltin & 0.60 & 0.609 \\
\hline Monooctyltin & 0.57 & 0.813 \\
\hline Dioctyltin & 0.57 & 0.752 \\
\hline
\end{tabular}

Table S11: Results of the paired Peto\&Peto generalized Wilcoxon test for differences between censored inlet and outlet substance concentrations, $\mathrm{n}=15$ pairs. Significant correlations, considering $\alpha=0.01$ are marked with a $*$ The test was only computed for substances for which $\mathrm{f}_{\text {censored }}<75 \%$. 


\section{Photographs of different types of sediments}

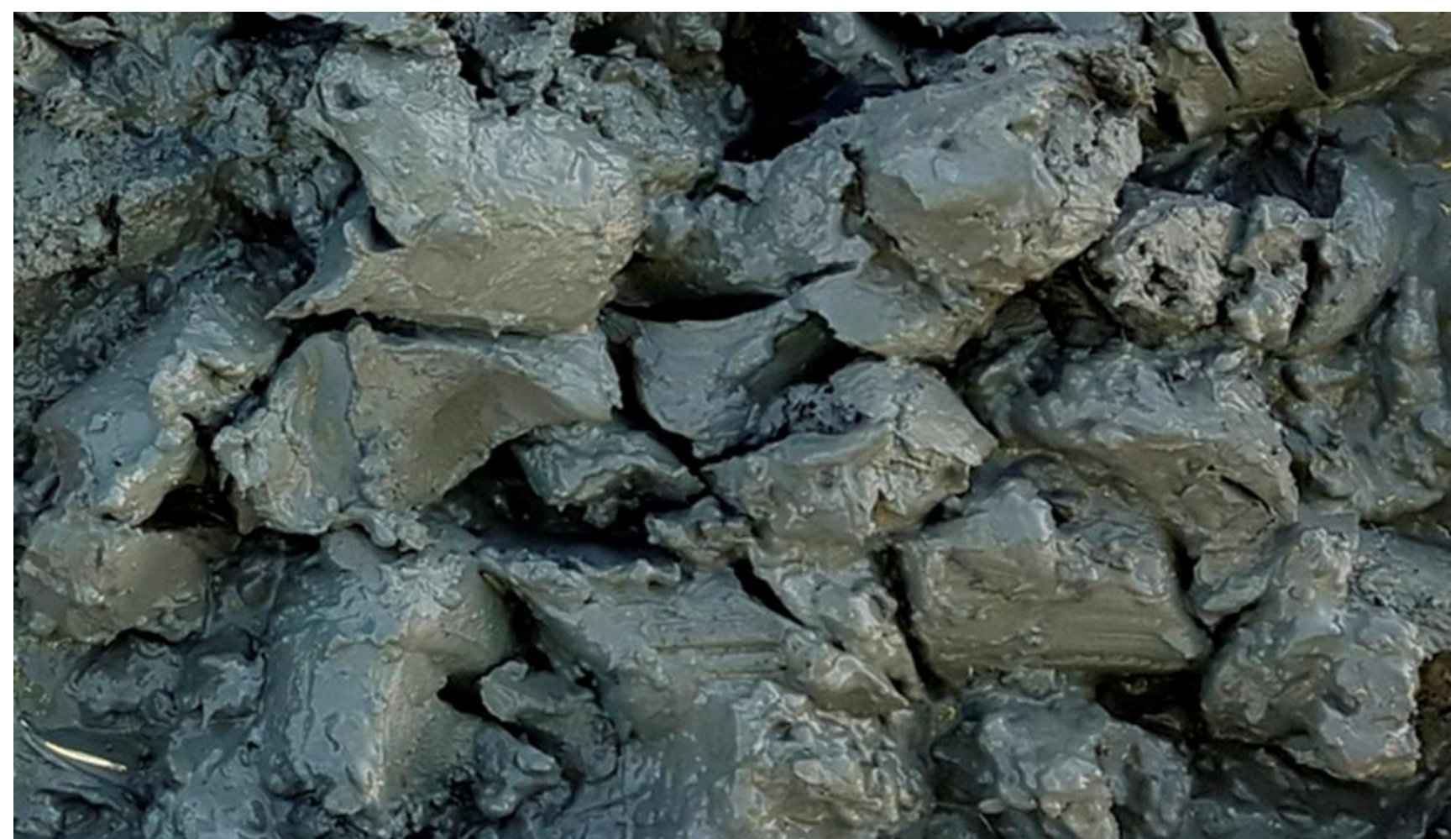

Figure S1a. Grey, clay sediments collected from pond Or-2

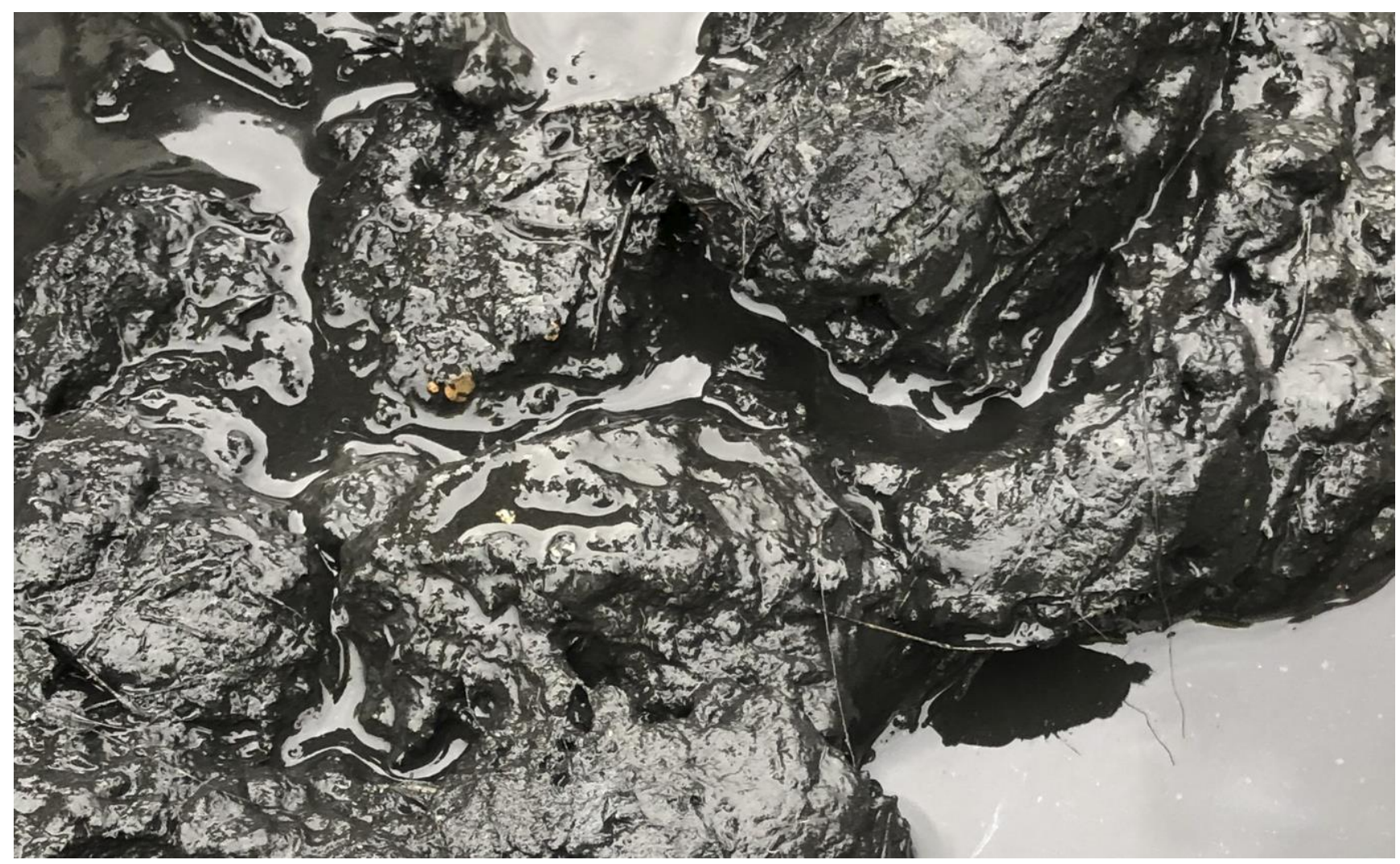

Figure S1b. Loose, black sediments collected from pond S-1. 


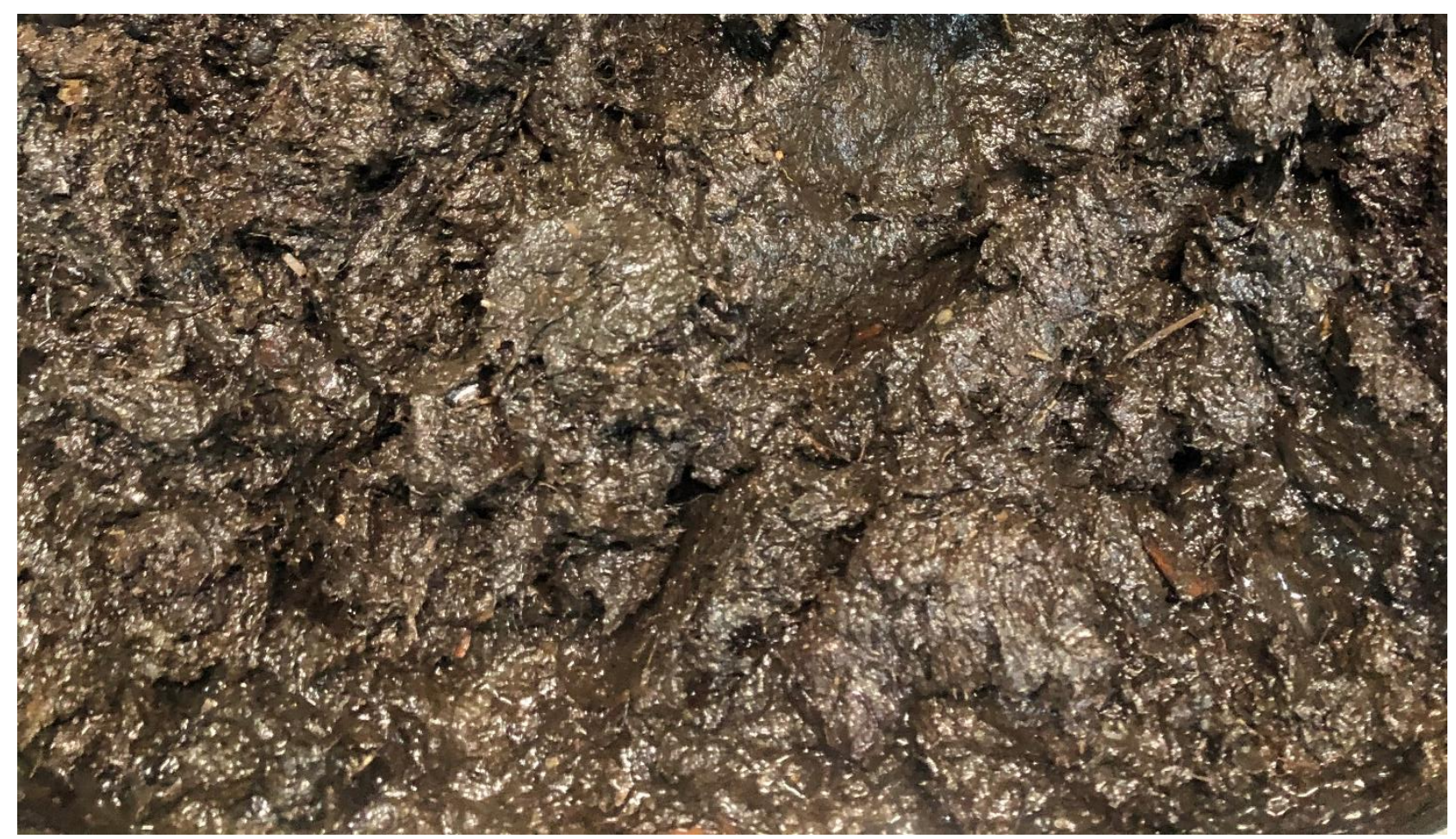

Figure S3c. Peat-like sediment collected from pond S-3

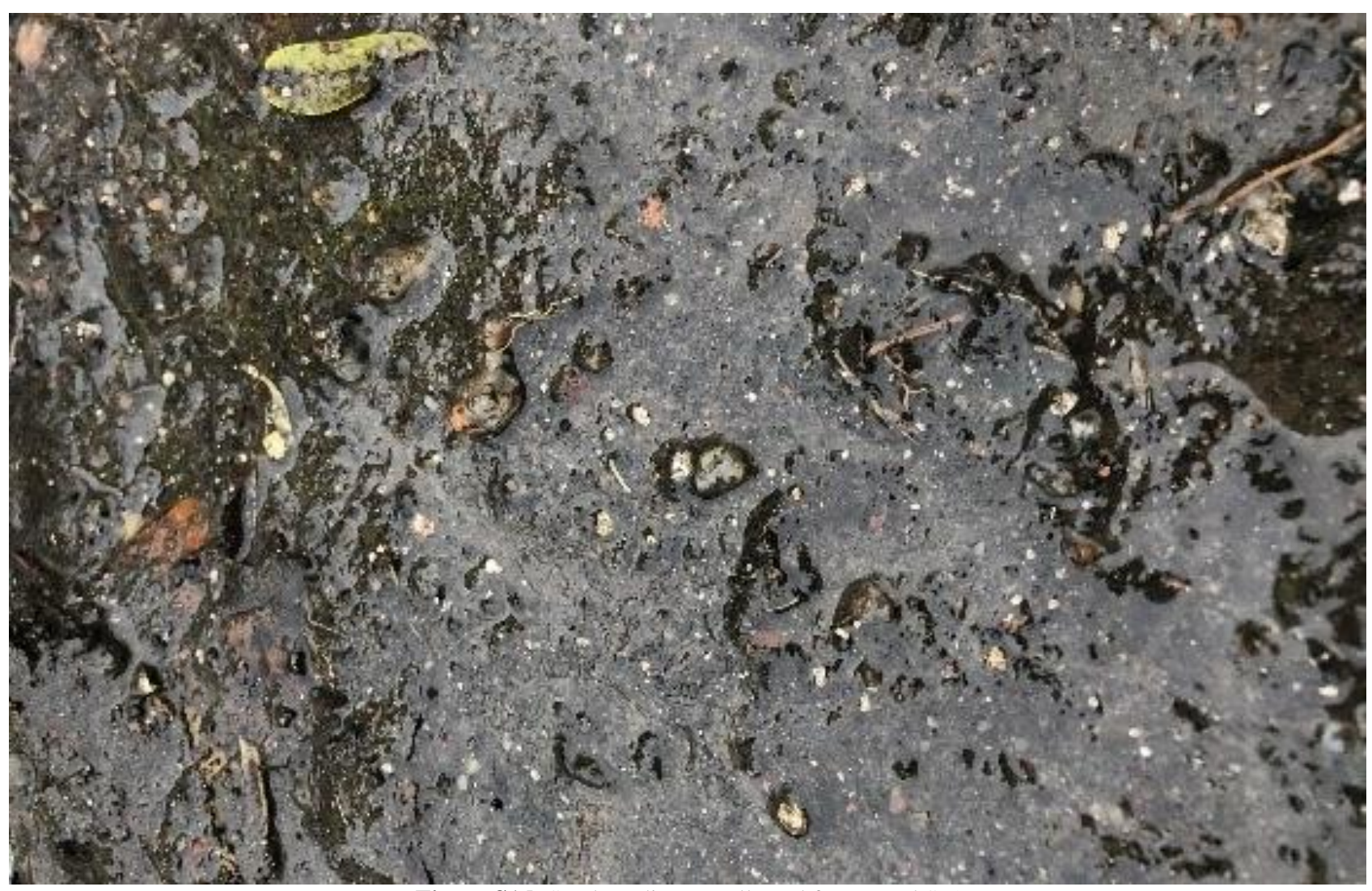

Figure S1d. Sandy sediment collected from pond S-5 
VIII. Regulatory limits exceeded by each sample

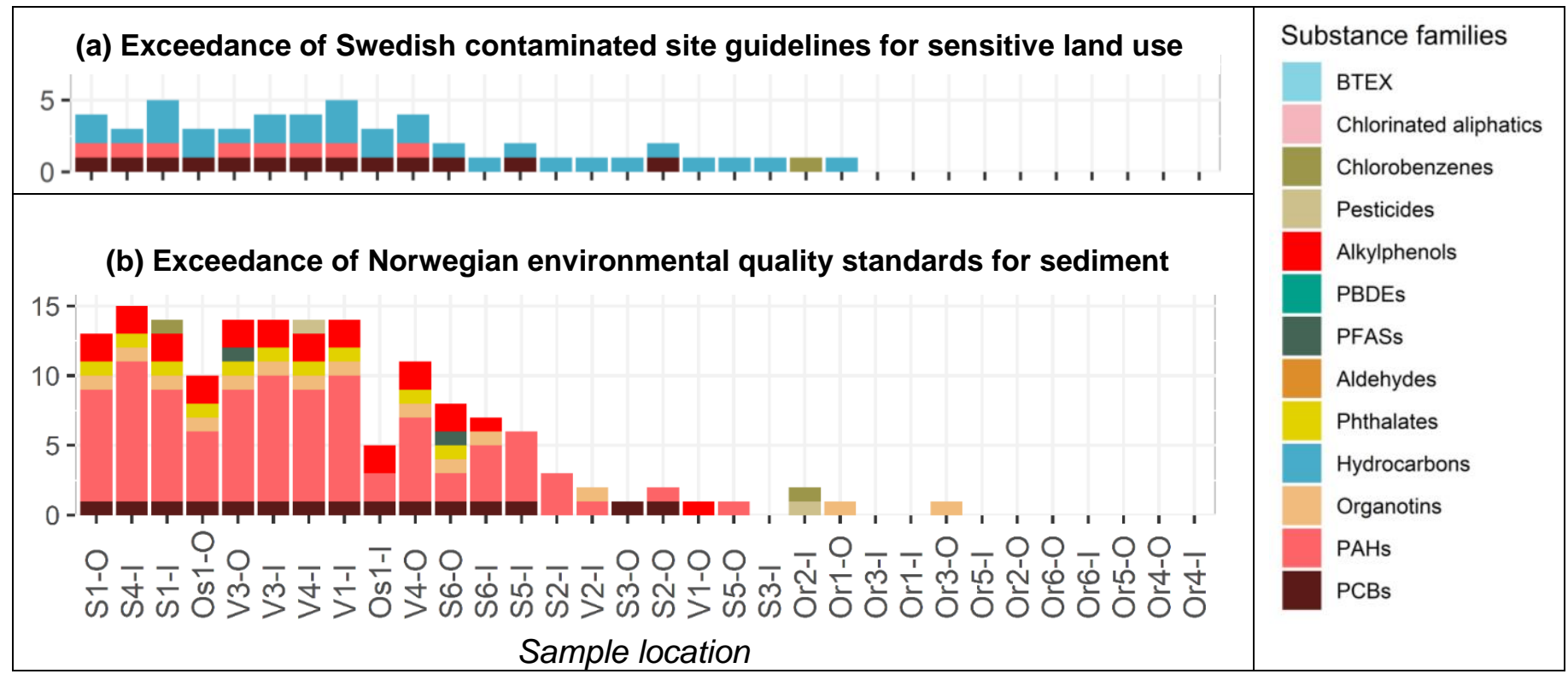

Figure S2a. Total number of quantified substances exceeding Swedish contaminated site guidelines for sensitive land use, ${ }^{50}$ and Figure S2b. total number of quantified substances exceeding Norwegian environmental quality standards for sediment ${ }^{51}$ 


\section{Ratios of observed concentrations to regulatory limits}

Note: The following graphs present the ratios of substance concentrations quantified in the stormwater sediments to corresponding environmental standards. Substances below the limit of quantification are not included in this graph. The two standard systems (Swedish contaminated site guidelines and Norwegian environmental quality standards for sediment) do not apply to the same lists of substances; in some cases, standards apply to several substances within a family.

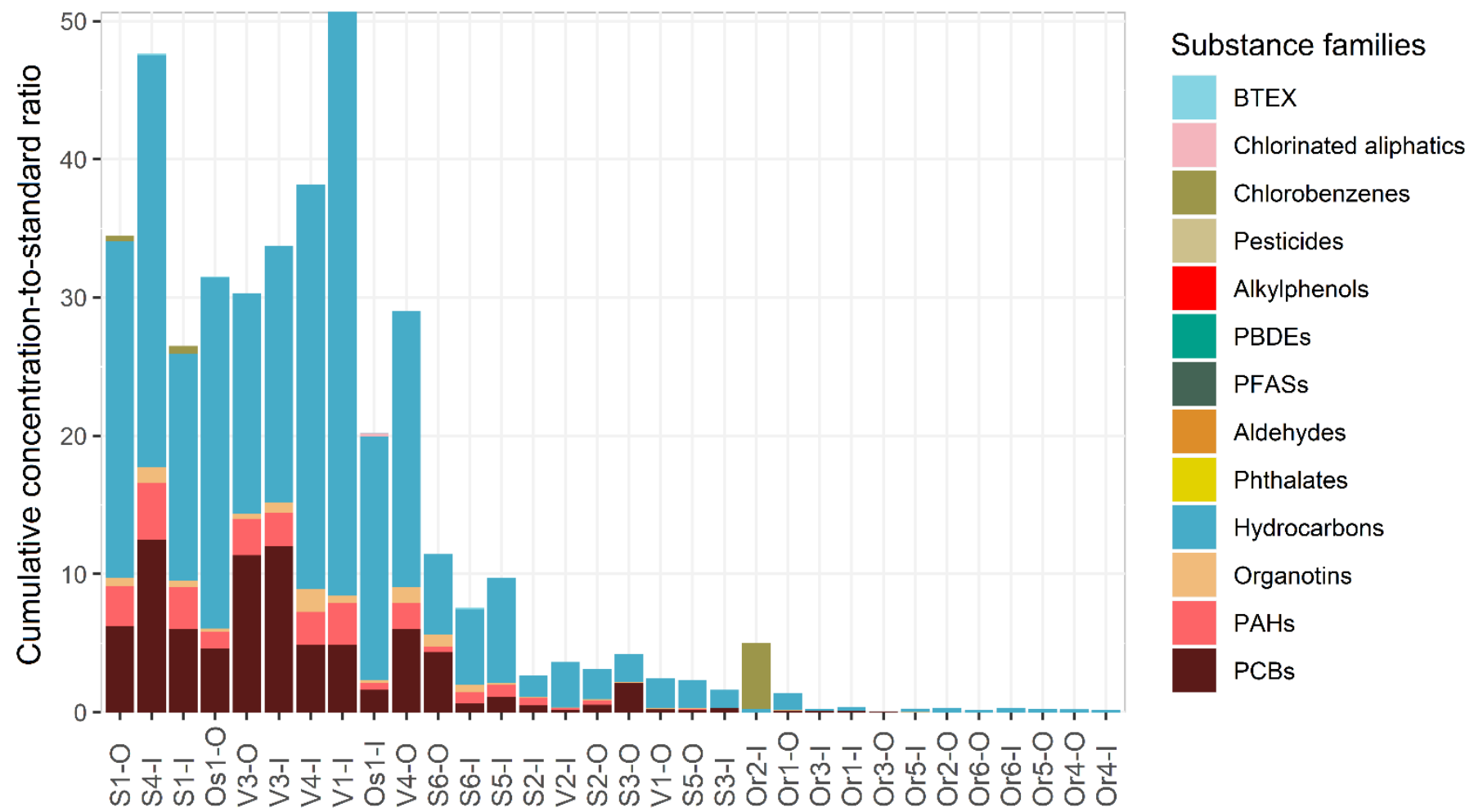

Figure S3a. Sum of ratios of quantified substance concentrations to Swedish contaminated site guidelines for sensitive land use, ${ }^{46}$ sorted by substance family. 


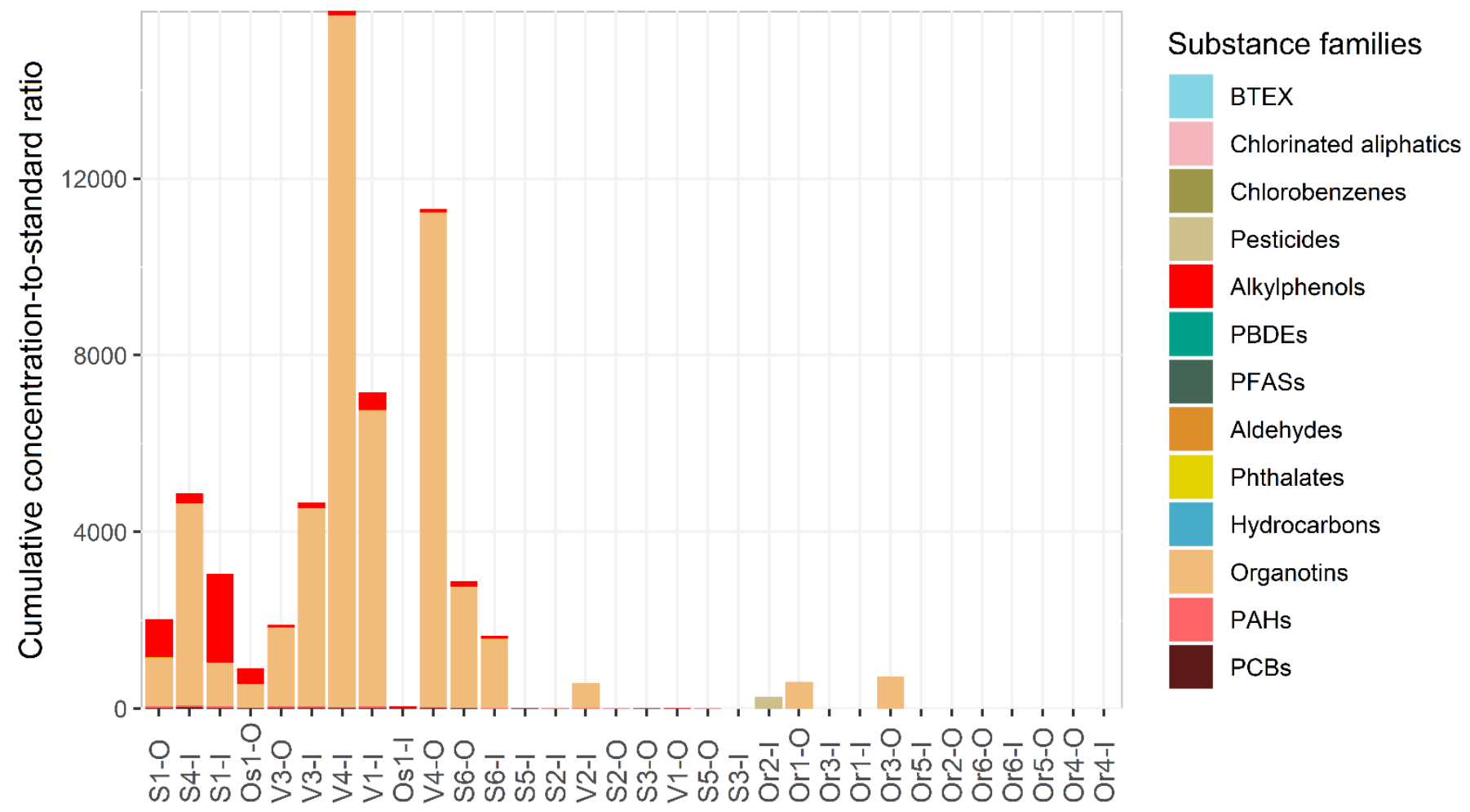

Figure S3b. Sum of ratios of quantified substance concentrations to Norwegian environmental quality standards for sediment ${ }^{47}$ sorted by substance family.

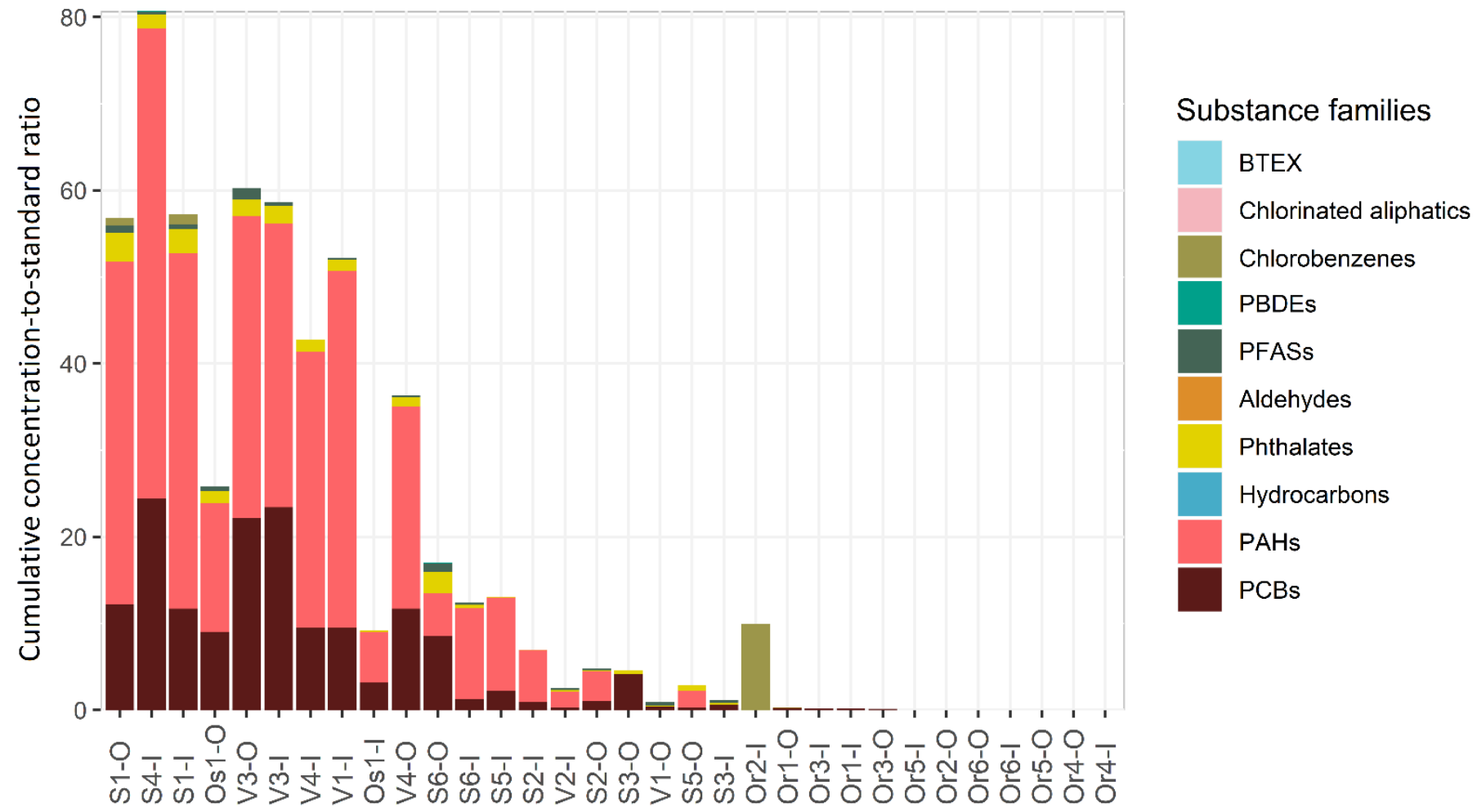

Figure S3c. Sum of ratios of quantified substance concentrations to Norwegian environmental quality standards for sediment ${ }^{47}$ sorted by substance family, excluding organotins, alkylphenols and pesticides 